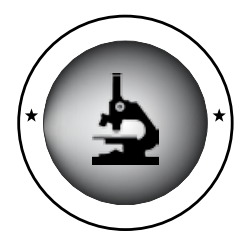

\title{
Congenital granular cell epulis: A rare diagnosis
}

\author{
Sigdel B ${ }^{1}$, Baidya $\mathrm{R}^{1}$, Shrestha $\mathrm{P}^{3}$, Shrestha $\mathrm{S}^{3}$ \\ ${ }^{1}$ Department of Pathology, B and B Hospital, Lalitpur, Nepal \\ ${ }^{2}$ Department of Dentistry, $B$ and B Hospital, Lalitpur, Nepal \\ ${ }^{3}$ Department of Paediatrics, $B$ and B Hospital, Lalitpur, Nepal
}

\section{Keywords: \\ Congenital; \\ Epulis; \\ Granular cell}

\begin{abstract}
Congenital granular cell tumor is very rare and it arises from the gum pad of neonates. Surgery is required immediatly after birth as the tumor interfares with feeding and respiration. Although a benign tumor, it can create immense anxiety amongst the attending physician and family members. Its recognition is important so as to avoid over diagnosis leading to radical surgery. We report a case of congenital epulis arising from the maxillary alveolar ridge in a newborn female child, which was successfully excised and the diagnosis confirmed histologically. No tumor recurrence has been reported in the patient postoperatively.
\end{abstract}

\section{INTRODUCTION}

Congenital epulis, also called congenital granular cell tumor of the newborn is a rare benign gingival cell tumor that presents at birth. The case was first described by Neumann in 1871. ${ }^{1,2,3}$ Two hundred and sixteen cases have been reported since then. ${ }^{1}$ The tumor is found in the alveolar ridges of neonates and occurs twice as often in the maxilla as in the mandible. ${ }^{1}$ Congenital epulis clinically appears as a pedunculated mass, which is typically protruding out of the newborn child's mouth and may interfere with feeding and respiration. The histogenesis is unknown ${ }^{3}$ and the tumor has a marked female preponderance. ${ }^{2}$ The presence of this lesion in the newborn can be traumatizing to the parents. Prompt surgical excision is the management of choice and has an excellent cure rate. Recurrence of lesion has not been reported to date. ${ }^{1}$ We report a case of congenital epulis in a newborn infant.

\section{Correspondence:}

Dr. Bandana Sigdel, $M D$

Department of Pathology, B and B Hospital, Gwarko, Lalitpur

E-mail: bandana_sigdel@hotmail.com

\section{REPORT}

A 24 year old primigravida underwent an elective caesarian section for maternal distress at 37 weeks of gestation. Over the previous 24 hours, she experienced shortness of breath, but did not have any significant past medical history and was not found to have any underlying medical disease. Ultrasound was done in the first, second and third trimesters which showed polyhydramnious, but no fetal abnormality was detected. A female child weighing $2.75 \mathrm{~kg}$ was born and the APGAR scores were 5, 6 and 9 at the $1^{\text {st }}, 5^{\text {th }}$ and $10^{\text {th }}$ minute respectively. She had a mass arising from the upper gum that protruded outside the mouth (fig. 1). The single mass had a slightly lobulated angiomatous appearance which was attached to the maxillary alveolar ridge. The mass prevented normal closure of the child's mouth, thus hindering normal breast or bottle feeding. The baby was put on nasogastric feeding. Airway was not obstructed. No other congenital abnormality was detected after thorough examination of the baby. The mass was excised on the $4^{\text {th }}$ post-operative day of lower segment cesarean section 


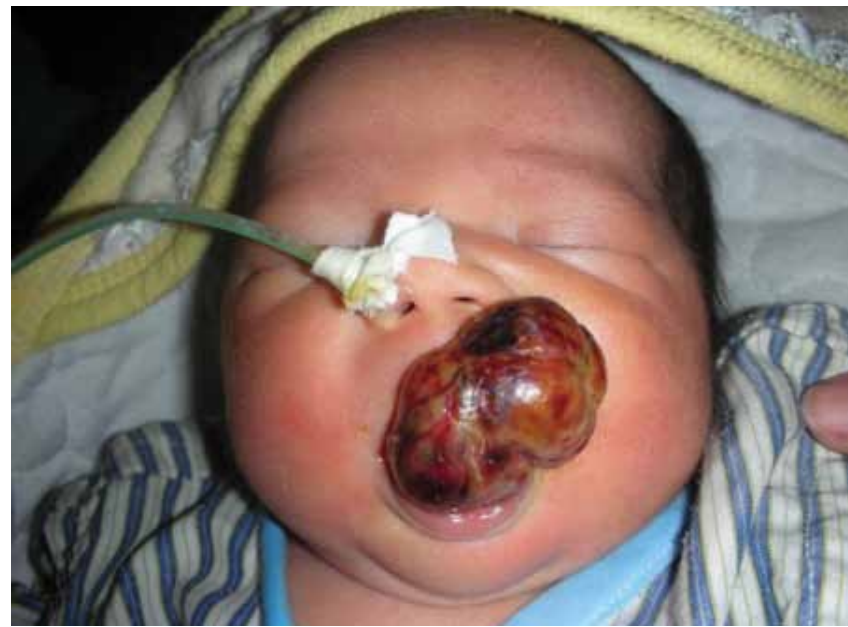

Figure 1: Newborn child with congenital epulis

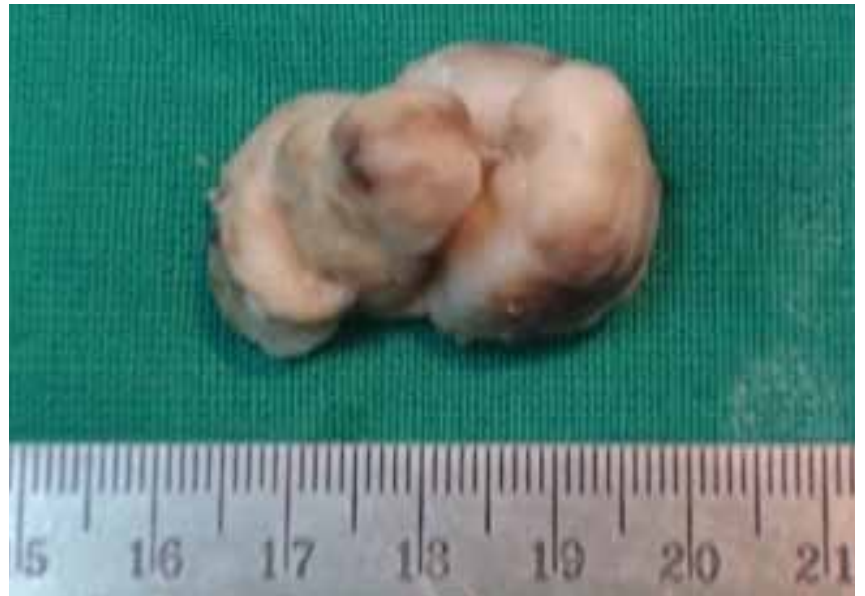

Figure 3: Gross specimen of the tumor .

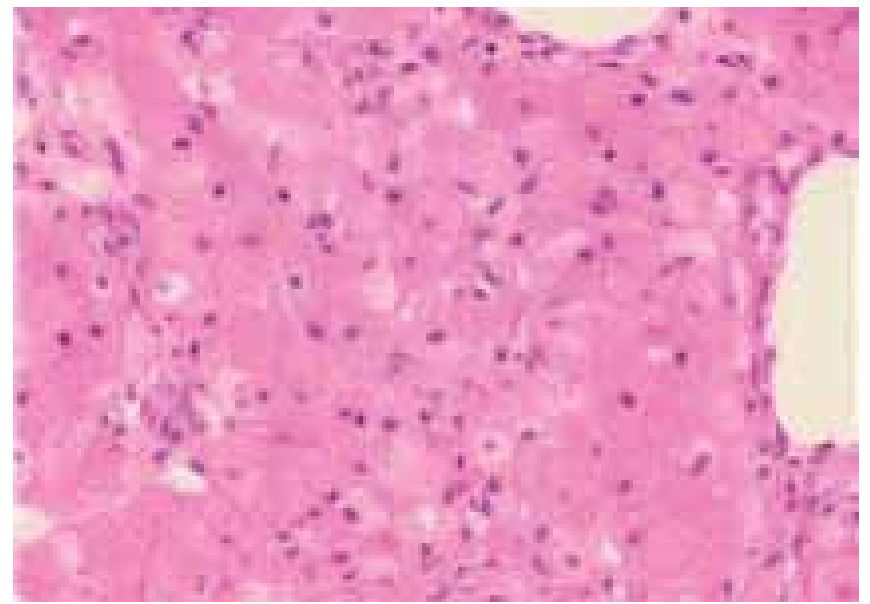

Figure 5: Microscopic feature of the tumor showing sheets of polyhedral cells with fine granular cytoplasm (HE stain, X10)

(LSCS) under local anaesthesia (fig. 2). No post-operative complication was encountered and bleeding was minimal. The site of incision healed within a few days. The child appeared completely normal, the nasogastric feeding was removed and the mother started feeding her normally. The sample was sent for histopathological evaluation which

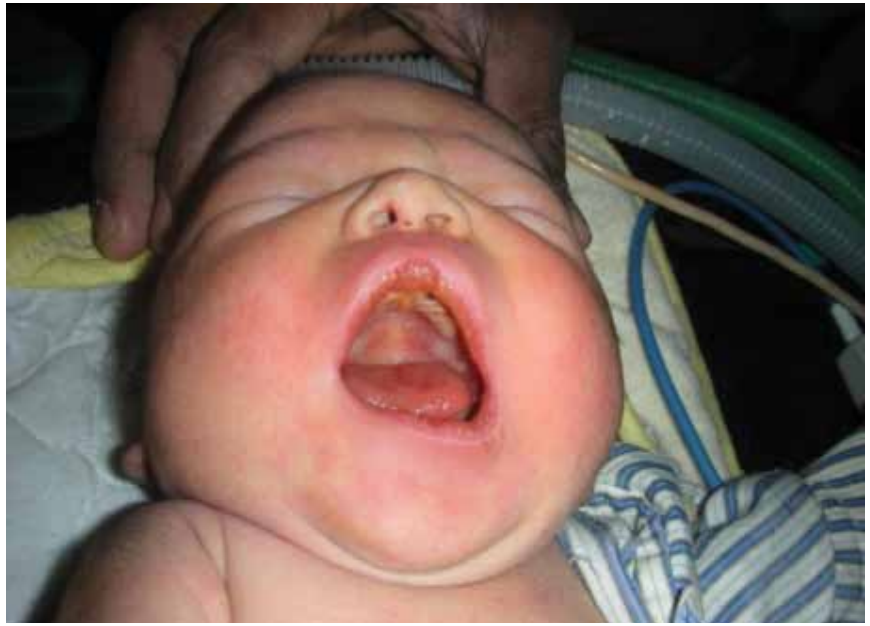

Figure 2: Child after surgery

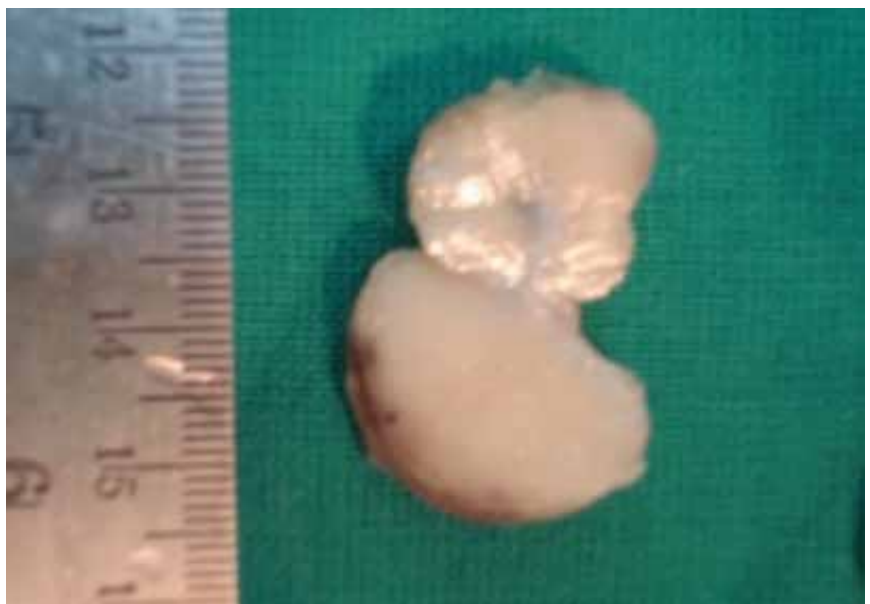

Figure 4: Cut surface of the tumor

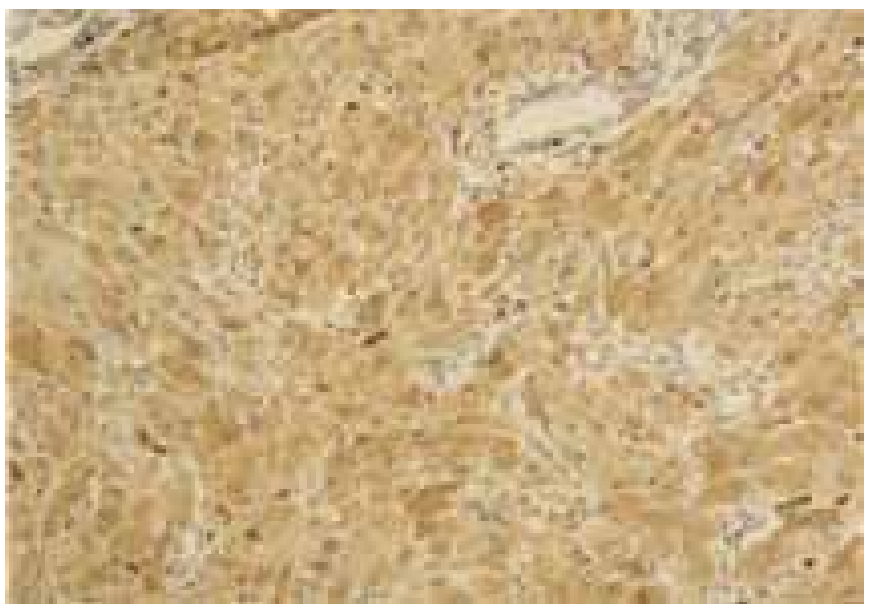

Figure 6: Microscopic picture of cells showing positivity for vimentin $(X 10)$

confirmed the diagnosis of a congenital granular cell tumor. Macroscopically, the tumor was brownish to grayish in color and had smooth but lobulated external surface and measured $4 \times 3 \times 2 \mathrm{~cm}$ (fig. 3). Cut surface was yellowish white colored, solid and homogenous. Histologically it was composed of solid sheets of large polyhedral cells with well- 
defined cell membrane, ample finely granular cytoplasm and bland nucleus without pleomorphism or mitotic activity (fig. 5). The lesion was well vascularized as evidenced by numerous thin walled slit like vessels. Focally, there was slight admixture with lymphocytic infiltrate. The overlying squamous epithelium was atrophic and, showed ulceration with deposition of fibrinogranulocytic exudate. The tumor cells were negative for Periodic acid-Schiff (PAS) stain. Immunohistochemical evaluation was as follows: Vimentin: Strongly positive (fig. 6), NSE: Positive, CD68: Positive. Cytokeratin, Desmin, CEA, ER, PR, S-100 protein: Negative.

The child was discharged and was on a regular follow-up on an outpatient basis. She was found to be completely healthy and there were no signs of relapse.

\section{DISCUSSION}

Congenital epulis is a rare benign ${ }^{1,4}$ gingival cell tumor that occurs on the gum pads of neonates. Since its first discovery in 1871 very few cases have been reported worldwide. This tumor has a close resemblance to granular cell myoblastoma. Epulis previously described for the lesion is derived from the Greek word and means "on the gums" or "gum boil". It arises from the mucosa of the gingiva and most commonly from the anterior part of the maxillary alveolar ridge. Maxillary predilection occurs in the ratio of 3:1. It often occurs as a single lesion, but in $10 \%$ of the cases the lesions are multiple. Our patient presented with a single lesion arising from the maxillary alveolar ridge. Eghbalian et.al. ${ }^{2}$ have described multiple tumors arising from the anterior maxillary alveolar ridge and Andreas Chiabi et al. have described the presence of two tumors in a newborn, each arising from the maxillary and mandibular mucosa. The tumor is known to have a marked female preponderance with a ratio of $8: 1 .^{2,5}$ The gender of our patient goes further to support the finding. The reason for female preponderance is not clear. A hormonal stimulus has been suspected but not proven since estrogen and progesterone receptors have not been found on the tumor to support the hypothesis. Immunohistochemistry for estrogen and progesterone receptors were negative.

The exact etiology of congenital epulis is unknown. ${ }^{1,2}$ Several theories have been proposed, namely myoblastic, odontogenic, neurogenic, histiocytic and endocrinologic, but the opinion of its pathogenesis lacks consensus. ${ }^{4}$ Immunohistochemically, as in most of the other reported cases, the tumor cells were positive for vimentin. In our cases and unlike other cases, NSE, a neural marker was positive. As in the case described by Lapid et al. ${ }^{5}$ they were also reactive for $\mathrm{CD} 68$. The tumor cells were negative for S-100 protein. Staining with PAS stain was negative. The pattern of immunostaining appeared diverse, contributing to the enigma of its histogenesis.
S-100 protein and PAS stain are positive in the usual granular cell tumor arising at the other sites in adults. Negativity for both of these supports congenital epulis as one of different cell origin and thus as a distinct entity. Presence at birth with predilection for newborn females, predominantly anterior maxillary location, lack of pseudoepitheliomatous hyperplasia and plexiform arrangement of capillaries are other distinguishing features.

No other related congenital abnormality was found in our patient. Prenatal obstretical Ultrasonogram (USG) failed to detect the abnormality. Charrier JB et al. ${ }^{7}$ have reported the detection of lesion at a 38-week prenatal ultrasound scan. Prenatal detection of the abnormality would be helpful for the parents and family members to become mentally prepared for the upcoming situation and for the medical team to receive and handle the child accordingly. Hence, modalities for prenatal detection of this type of lesions need to be developed more in future.

Histopathological evaluation of the tumor is important to confirm the diagnosis. This will decrease the anxiety for everyone related to the case and justify avoiding radical surgery. It is a very distressing experienced for the parents to find their child at birth to have a mass protruding through the mouth. The social and esthetic problems arising from the situation is also great in a country like Nepal where superstitions still prevail.

\section{CONCLUSION}

Congenital epulis, which presents as a benign mass protruding through the mouth of a newborn baby, can be very frightening to the parents. When the tumor is large and obstructive, it can impair feeding and respiration. A simple surgical resection is curative. Awareness about this condition can prevent over diagnosis and unnecessary extensive surgery.

\section{ACKNOWLEDGEMENT}

I would like to express my sincere thanks to Prof. F. T. Bosman from the Department of Pathology, University of Laussane, for his immense help in working up the case.

\section{REFERENCES}

1. Reinshagen K, Wessel LM, Roth H, Waag KL. Congenital epulis: a rare diagnosis in padiatric surgery. Eur J Pediatr Surg 2002;12:124-6.

2. Eghbalian F, Monsef A; Congenital epulis in the newborn, review of the literature and a case report. J Pediatr Hematol Oncol 2009;31:1989.

3. Mabongo M, Wood NH, Lemmer J, Feller L. Congenital epulis. A case report. SADJ. 2008;63:350-1.

4. Oslon JL, Marcus JR, Zuker RM. Congenital epulis. J Craniofac Surg 2005; 16:161-4.

5. Lapid O, Shaco-Levy R, Krieger Y, Kachko L, Sagi A. Congenital Epulis. Pediatrics 2001;107:E22. 
6. Bilen BT, Alaybeyoglu N, Aslan S, Turkmen E, Celik M. Obstructive congenital gingival granular cell tumor. Int J Pediatr Otorhinolaryngol 2004;68:1567-71.
7. Charrier JB, Droulle P, Vignaud JM, Chassagne JE, Stricker M. Obstructive congenital gingival granular cell tumor. Ann Otol Rhinol Laryngol 2003;112:388-91. 